\title{
Peptide 11R-VIVIT promotes fracture healing in osteoporotic rats
}

\author{
CHANGJU HOU, XUEPENG WANG, WU JIANG, ZHENYU BIAN, LIULONG ZHU and MAOQIANG LI \\ Department of Orthopedics, The Affiliated Hangzhou First People's Hospital, \\ Zhejiang University School of Medicine, Hangzhou, Zhejiang 310006, P.R. China
}

Received December 8, 2020; Accepted March 31, 2021

DOI: $10.3892 /$ ijmm.2021.4995

\begin{abstract}
Osteoporotic fracture healing is a complex clinical issue. The present study was conducted to investigate the repair properties of 11R-VIVIT on osteoporotic fractures and to examine the potential effects of 11R-VIVIT on osteoporotic bone marrow-derived mesenchymal stem cells (BMSCs), A rat model of osteoporotic femoral fracture was established, and the effects of the daily local injection of 11R-VIVIT or saline on fracture repairing were evaluated by micro-CT scans and $\mathrm{H} \& \mathrm{E}$ staining. Moreover, BMSCs from osteoporotic rats were treated with 11R-VIVIT, and the osteogenic and adipogenic differentiation of BMSCs was evaluated. The results revealed that 11R-VIVIT promoted bone formation and increased fracture healing. In addition, 11R-VIVIT promoted the differentiation of osteoporotic BMSCs into osteoblasts rather than adipocytes. Furthermore, mechanistic analysis revealed that 11R-VIVIT promoted autophagy by blocking the protein kinase B (AKT)/nuclear factor of activated T-cells (NFATc1) signaling pathway. Consistently, the activation and inhibition of autophagy using rapamycin and LY294002 confirmed the regulatory effects of 11R-VIVIT on autophagy. On the whole, the findings of the present study demonstrate that 11R-VIVIT promotes fracture healing in osteoporotic rats and enhances the osteogenic differentiation of osteoporotic BMSCs by dysregulating the AKT/NFATc1 signaling pathway.
\end{abstract}

Correspondence to: Dr Maoqiang Li, Department of Orthopedics, The Affiliated Hangzhou First People's Hospital, Zhejiang University School of Medicine, 261 Huansha Road, Hangzhou, Zhejiang 310006, P.R. China

E-mail: limaoqiangzhong@163.com

Abbreviations: BMSCs, bone marrow-derived mesenchymal stem cells; NFAT, nuclear factor of activated T-cells; AKT, protein kinase B; GSK-3 $\beta$, glycogen synthase kinase $3 \beta$; Cn, calcineurin; OIM, osteogenic induction medium; AIM, adipogenic induction medium; ALP, alkaline phosphatase; PCR, polymerase chain reaction; OCN, osteocalcin; SDS-PAGE, sodium dodecyl sulfate polyacrylamide gel electrophoresis

Key words: osteoporotic fracture healing, 11R-VIVIT, NFATc1, BMSCs

\section{Introduction}

Osteoporosis is the most prevalent systemic skeletal system disease, leading to increased bone fragility and vulnerability to fractures $(1,2)$. Due to the microarchitectural destruction in bone tissue, fracture healing in osteoporotic patients is often delayed and compromised compared with non-osteoporotic individuals $(3,4)$. Osteoporosis usually results from menopause, aging, metabolic diseases and drug therapies with the precise cellular and molecular mechanism remaining to be elucidated $(2,5)$.

Currently, dysregulated bone turnover, particularly the dysregulated differentiation of bone marrow-derived mesenchymal stem cells (BMSCs) has been discovered as an important factor of osteoporotic fractures $(6,7)$. In detail, the osteoblastic differentiation of BMSCs is impaired, with a greater number of BMSCs being differentiated into adipocytes in osteoporotic patients, leading to progressive dysregulated bone turnover and bone loss $(5,6)$. Therefore, approaches for fracture healing based on the activation of the osteogenic differentiation and the inhibition of the adipogenic differentiation of BMSCs are innovative and appealing $(8,9)$.

The nuclear factor of activated T-cells (NFAT) is a substrate of the $\mathrm{Ca}^{2+}$-dependent transcription factor family, which has been shown to regulate cell differentiation and organ development, podocyte injury and cell apoptosis. In addition, NFAT signaling has been found to play critical roles in bone metabolism, such as in the process of osteoblast and osteoclast formation (10). In detail, the activation of the NFAT signaling pathway has been shown to lead to the inhibition of osteoprogenitor cell formation (11). By contrast, the blocking of NFAT signaling will contribute to the osteoblastic differentiation of MSCs $(11,12)$, which suggests that NFAT is a potential target for the treatment of osteoporotic fractures. Peptide 11R-VIVIT is a NFAT-specific inhibitor without affecting upstream calcineurin (Cn). Due to its high specificity against NFAT2, peptide 11R-VIVIT has been widely examined and few adverse effects have been reported (13-15). A Previous study demonstrated that 11R-VIVIT can stimulate bone formation by decreasing NFATc1 expression and regulating the expression of inflammation-related molecules (10). However, whether peptide 11R-VIVIT can affect the healing process of osteoporotic fractures via the downregulation of NFATc1 remains unknown.

The present study aimed to elucidate the effects and underlying molecular mechanisms of peptide 11R-VIVIT in fracture 
healing. The results indicated that $11 \mathrm{R}-\mathrm{VIVIT}$ promoted autophagy to enhance the osteogenic differentiation of osteoporotic BMSCs to promote fracture healing in osteoporotic rats through the protein kinase $\mathrm{B}(\mathrm{AKT}) / \mathrm{NFATc1}$ signaling pathway, which may provide new insight for the BMSC-based treatment of osteoporosis in the future.

\section{Materials and methods}

Animals and ethics statement. Following the Animal Experimentation Ethics Guidelines, the Ethics Committee of the Affiliated Hangzhou First People's Hospital approved all the procedures in the study (no. ZJCLA-IACUC-20080010). A total of 24 Sprague-Dawley female rats (12 weeks old, weighing 200-240 g) were purchased from the Beijing Weitong Lihua Experimental Animal Technology Co. Ltd. (Beijing, China). Then, the rats were housed in a specific pathogen-free (SPF) facility (temperature, $22 \pm 2^{\circ} \mathrm{C}$; humidity, $50 \pm 10 \%$ ) with a 12/12-h light/dark cycle, and standard laboratory animal chow and tap water were available ad libitum. To assess the effects of peptide 11R-VIVIT on osteoporotic fracture healing, a rat model of osteoporosis [model of ovariectomy (OVX)-induced osteoporosis] was established at first by removing the ovaries bilaterally (16). At 12 weeks after the OVX surgery, a unilateral open femur fracture model was established in all rats by an experienced operator to reduce pain in the animals. Briefly, an osteotomy fracture was established using an oscillating sagittal saw the middle of the left femur and 25-G needles were inserted intramedullary for fixation. A total of four groups $(n=6$ for each group) were included in the study as follows: i) A group of whole-stage saline treatment (Saline/Saline group, saline treatment only during ovariectomy and sacrifice); ii) a group of late-stage VIVIT (intraperitoneal injection, $100 \mu \mathrm{g} / \mathrm{kg}$, Tocris Bioscience) treatment (Saline/VIVIT group, saline treatment following OVX and 11R-VIVIT treatment following OVX); iii) a group of early-stage 11R-VIVIT following saline treatment (VIVIT/Saline group, 11R-VIVIT treatment following OVX and saline treatment following OVX); and iv) a group of whole-stage 11R-VIVIT treatment (VIVIT/VIVIT group, 11R-VIVIT treatment only during OVX and sacrifice). Food and water were provided without limits to all rats during the fracture healing process. All animals were anesthetized by an intraperitoneal injection $(50 \mathrm{mg} / \mathrm{kg})$ of $1 \%$ sodium pentobarbital during the surgeries. The rats were then euthanized by cervical dislocation under anesthesia. The femurs were collected for X-ray, micro-CT and histological analyses. All wounds healed well and no death or adverse events were observed at the experimental endpoint.

$X$-ray and micro-CT evaluation and histological staining. Femurs were fixed in $4 \%$ paraformaldehyde for $24 \mathrm{~h}$ and then scanned with an X-ray scanner or a micro-CT system. The region of interest was defined as $2 \mathrm{~mm}$ above and below the fracture. All the slices derived from the micro-CT scanning were collected for $3 \mathrm{D}$ reconstruction. The bone formation area, including callus and cortex bone around the fracture line was selected to measure fracture healing. Following X-ray and micro-CT evaluation, the femurs were decalcified using EDTA, sliced into $4-\mu \mathrm{m}$-thick sections and stained with hematoxylin and eosin (Sigma-Aldrich; Merck KGaA) at room temperature for $30 \mathrm{~min}$. Histological images were observed under a microscope (XSP-C204, China Investment Corporation).

Isolation of rat BMSCs. The BMSCs were isolated and obtained by referring to a previous study (17). In brief, after the rats were anesthetized, the rat tibia was separated and obtained. The ends of the rat tibia were cut with scissors, and the tibia bone marrow contents were flushed and washed with phosphate-buffered saline (PBS) supplemented with 5\% fetal bovine serum (FBS). Thereafter, the bone marrow contents were centrifuged at $450 \mathrm{x} \mathrm{g}$ for $10 \mathrm{~min}$ at room temperature and the cell pellet was re-suspended, which was then layered using the same volume of Ficoll-Paque (inno-train Diagnostik $\mathrm{GmbH}$ ) and centrifuged at $850 \mathrm{~g}$ for $25 \mathrm{~min}$ at $4^{\circ} \mathrm{C}$. Subsequently, BMSCs was collected and re-suspended in $\alpha$-MEM containing $10 \%$ FBS and $1 \%$ penicillin-streptomycin (all from HyClone; Cytiva). Cell cultures were maintained in a $37^{\circ} \mathrm{C}$ incubator with $5 \% \mathrm{CO}_{2}$, and the medium was replaced twice weekly in the present study. Cells at passage 3-6 were utilized throughout the present study.

Characterization of BMSCs and multi-lineage differentiation. Flow cytometric analysis for the characterization of BMSCs was conducted by referring to the method described in previous studies $(18,19)$. Briefly, $1 \times 10^{6}$ BMSCs were incubated with (FITC or PE)-conjugated antibody CD45 (cat. no. 559135, BD Biosciences), CD11b (cat. no. 554982, BD Biosciences), CD73 (cat. no. 11-0739-42, Invitrogen; Thermo Fisher Scientific, Inc.) and CD90 (cat. no. 554898, BD Biosciences) $\left(1 \mu \mathrm{g} / 10^{6}\right.$ cells) in washing buffer for $30 \mathrm{~min}$ at $4^{\circ} \mathrm{C}$. All fluorescently-labeled antibodies used in flow cytometric analysis were purchased from BD Biosciences. Thereafter, both the stained and unstained cells were washed, and their fluorescence intensity was quantified using a FACSCalibur flow cytometer (BD Biosciences). FlowJo software (Ver. 6.2) was utilized for data analysis.

BMSCs were pre-treated with $0.1,1$ and $10 \mu \mathrm{M} 11 \mathrm{R}-\mathrm{VIVIT}$, $10 \mu \mathrm{M}$ LY294002 (Sigma-Aldrich; Merck KGaA) and $200 \mathrm{nM}$ rapamycin (Beijing Solarbio Science \& Technology Co., Ltd.) for $1 \mathrm{~h}$.

To detect the osteogenic ability, BMSCs were inoculated at approximately $1 \times 10^{4}$ cells $/ \mathrm{cm}^{2}$ in 24 -well plates and induced in osteogenic induction medium (OIM; cat. no. RASMX-90021, Cyagen Biosciences). Likewise, commercial standard adipogenic induction medium (AIM; cat. no. RASMX-90031, Cyagen Biosciences,) was employed for the induction of adipogenesis, which revealed the adipogenic ability of the BMSCs.

ALP staining and ALP activity assays were performed using BMSCs that had been cultured in OIM for 7 days. For ALP staining, BMSCs were washed with PBS, fixed in $4 \%$ paraformaldehyde for $20 \mathrm{~min}$ at room temperature, and then stained with an ALP staining kit (Nanjing Jiancheng Bioengineering Institute) for $30 \mathrm{~min}$ at room temperature in the dark. For ALP activity quantification, osteogenically differentiated BMSCs were examined using an EscAPeTM SEAP Chemiluminescence kit (Clontech Laboratories, Inc.), the value of which was normalized to the total cellular protein concentrations. Alizarin Red S staining and Oil Red O staining were performed at day 14 following differentiation. For Alizarin Red S staining, BMSCs were fixed with $4 \%$ paraformaldehyde 
for $20 \mathrm{~min}$, washed PBS, and then stained with Alizarin Red (Cyagen Biosciences) for $5 \mathrm{~min}$ at room temperature. Images obtained following staining were analyzed using Image J software (ver. 1.6, Wayne Rasband, National Institutes of Health) and the Alizarin Red S percentage of the positively stained area was determined. Finally, Oil Red O staining was applied to determine the adipogenic ability of the BMSCs. In brief, BMSCs were fixed in $3.7 \%$ formaldehyde for $30 \mathrm{~min}$, followed by staining with Oil Red O (Sigma-Aldrich; Merck KGaA) for 30 min. All images were acquired using a light microscope (Dmi8, Leica Microsystems GmbH).

Reverse transcription and quantitative polymerase chain reaction $(R T-q P C R)$ and semi-quantitative $P C R$. Total RNA was extracted from the BMSCs with TRIzol ${ }^{\circledR}$ reagent (Invitrogen; Thermo Fisher Scientific, Inc.). Reverse transcription was carried out using the Script ${ }^{\mathrm{TM}}$ RT-PCR kit (Takara Bio, Inc.), followed by PCR using SYBR PreMix Ex TaqTM (Takara Bio, Inc.) on the CFX96TM Real-time PCR Detection System (Applied Biosystems). GAPDH mRNA was used as an internal control. The reaction conditions were as follows: $95^{\circ} \mathrm{C}$ for $30 \mathrm{sec}$, followed by 40 cycles of $94^{\circ} \mathrm{C}$ for $5 \mathrm{sec}$ and $60^{\circ} \mathrm{C}$ for $35 \mathrm{sec}$. The $2^{-\Delta \Delta \mathrm{Cq}}$ method (20) was used to determine the relative expression levels. The primers for rat mRNAs are listed in Table I. The amplified products were separated on $1 \%$ agarose gel stained with ethidium bromide and visualized by UV light

Western blot analysis. Western blot analysis was carried out by referring to the protocol described in a previous study (21). Total proteins were isolated from the BMSCs using RIPA-lysis buffer (Sigma-Aldrich; Merck KGaA) on ice, and protein concentrations were measured using a BCA protein assay kit (Sigma-Aldrich; Merck KGaA). A total of $20 \mu \mathrm{g}$ of total protein were electrophoresed on 12\% SDS-PAGE and transferred to nitrocellulose membranes. The membranes were blocked using non-fat milk for $1 \mathrm{~h}$ at room temperature and incubated overnight at $4^{\circ} \mathrm{C}$ with primary polyclonal antibodies including anti-LC3 (ab192890, 1:2,000), anti-p62 (ab109012, 1:10,000), anti-p-AKT (ab38449, 1:1,000), anti-AKT (ab8805, 1:500), anti-p-glycogen synthase kinase-3 $\beta$ (GSK-3 $\beta$, ab131097, 1:1,000), anti-GSK-3 $\beta$ (ab93926, 1:1,000), anti-NFATc1 (ab25916, 1:2,000), anti-Lamin B (ab32532, 1:500), anti-Runt-related transcription factor 2 (Runx2; ab76956, 1:2,000) (all from Abcam), anti-ALP (PA1004, 1:1,000, Boster Biological Technology Co., Ltd.) and anti-GAPDH (ab8245, 1:1,000, Abcam). The proteins were then visualized following incubation with goat anti-rat $\mathrm{IgG}(\mathrm{H} \& \mathrm{~L})$ secondary antibody (ab182018, 1:2,000, Abcam) for $60 \mathrm{~min}$ at $25^{\circ} \mathrm{C}$, with extensively washing with TBST and detecting using a chemiluminescence system (Pierce; Thermo Fisher Scientific, Inc.). The intensity of protein bands was measured by ImageJ 1.6 software and the signal intensity of each band was normalized to its corresponding GAPDH or Lamin B control.

CCK-8 assay. The viability of BMSCs was measured using a CCK-8 kit (Dojindo Molecular Technologies, Inc.). In detail, normal BMSCs, osteoporotic BMSCs and osteoporotic BMSCs treated with 11R-VIVIT $(10 \mu \mathrm{M})$ in 96-well plates were collected at the time points of $0,24,48$ and $72 \mathrm{~h}$. CCK- 8 kit solution $(15 \mu \mathrm{l})$ were added to each well, and the cells were
Table I. Sequences of primers used for PCR.

\begin{tabular}{ll} 
Gene & \multicolumn{1}{c}{ Primer pair sequence (5'-3') } \\
\hline ALP & AAGGCTTCTTCTTGCTGGTG \\
OCN & GGCAGCACCCTCATGATGTCC \\
& CTGGAGAGGAGCAGAACTGG \\
OPN & CATCAGAGCCACGAGTTTCA \\
PPAR $\gamma$ & TCAGGGCCCAAAACACTATC \\
& GGCTTCATGACAAGGGAGTTTC \\
LPL & AACTCAAACTTGGGCTCCATAAAG \\
& CTGGACGGTAACAGGAATGTATGAG \\
GAPDH & CATCAGGAGAAAGACGACTCGG \\
& TCGACAGTCAGCCGCATCTTCTTT \\
& GCCCAATACGACCAAATCCGTTGA
\end{tabular}

ALP, alkaline phosphatase; OCN, osteocalcin; OPN, osteopontin; $\operatorname{PPAR} \gamma$, peroxisome proliferator-activated receptor $\gamma$; LPL, lipoprotein lipase.

incubated at $37^{\circ} \mathrm{C}$ for an additional $3 \mathrm{~h}$. The solution was finally removed, and the absorbance at $450 \mathrm{~nm}$ was recorded using a microplate spectrophotometer (Bio-Tek Instruments, Inc.).

Enzyme-linked immunosorbent assay (ELISA). The tumor necrosis factor (TNF)- $\alpha$ and interleukin (IL)- $1 \beta$ concentrations of the normal BMSCs, osteoporotic BMSCs and osteoporotic BMSCs treated with 11R-VIVIT $(10 \mu \mathrm{M})$ were measured by ELISA. The cells were cultured in 96-well plates for 3 days and the culture media were harvested and centrifuged at $1,200 \mathrm{xg}$ for $10 \mathrm{~min}$ at room temperature. The TNF- $\alpha$ and IL-1 $\beta$ concentrations in the supernatants were detected using rat TNF- $\alpha$ (EK0526) and IL-1 $\beta$ (EK0393) ELISA kits (Boster Biological Technology Co., Ltd.), according to the standard instructions. The value at $450 \mathrm{~nm}$ was read using a microplate reader (SpectraMax 190, Molecular Devices, LLC). The concentrations were calculated using the standard curve.

Statistical analysis. All data were presented as the mean \pm SD. Statistical analyses were calculated using GraphPad Prism (version 8.0) software. The Student's t-test was adopted for two-sample comparisons. One-way ANOVA followed by Tukey's post hoc analysis were adopted for multiple comparisons. All data were determined by at least three independent experiments. $\mathrm{P}<0.05$ was considered to indicate a statistically significant difference.

\section{Results}

$11 R$-VIVIT accelerates fracture healing. To determine whether the model of OVX was successfully established, bone mineral density in the rats was detected. The results revealed that rats subjected to OVX had a lower bone mineral density than the rats at baseline (Fig. 1A). Thereafter, the effects of 11R-VIVIT on the osteoporotic fracture healing process were examined (Fig. 1B). Micro-CT scans results revealed that the rats treated with 11R-VIVIT had a higher bone volume 

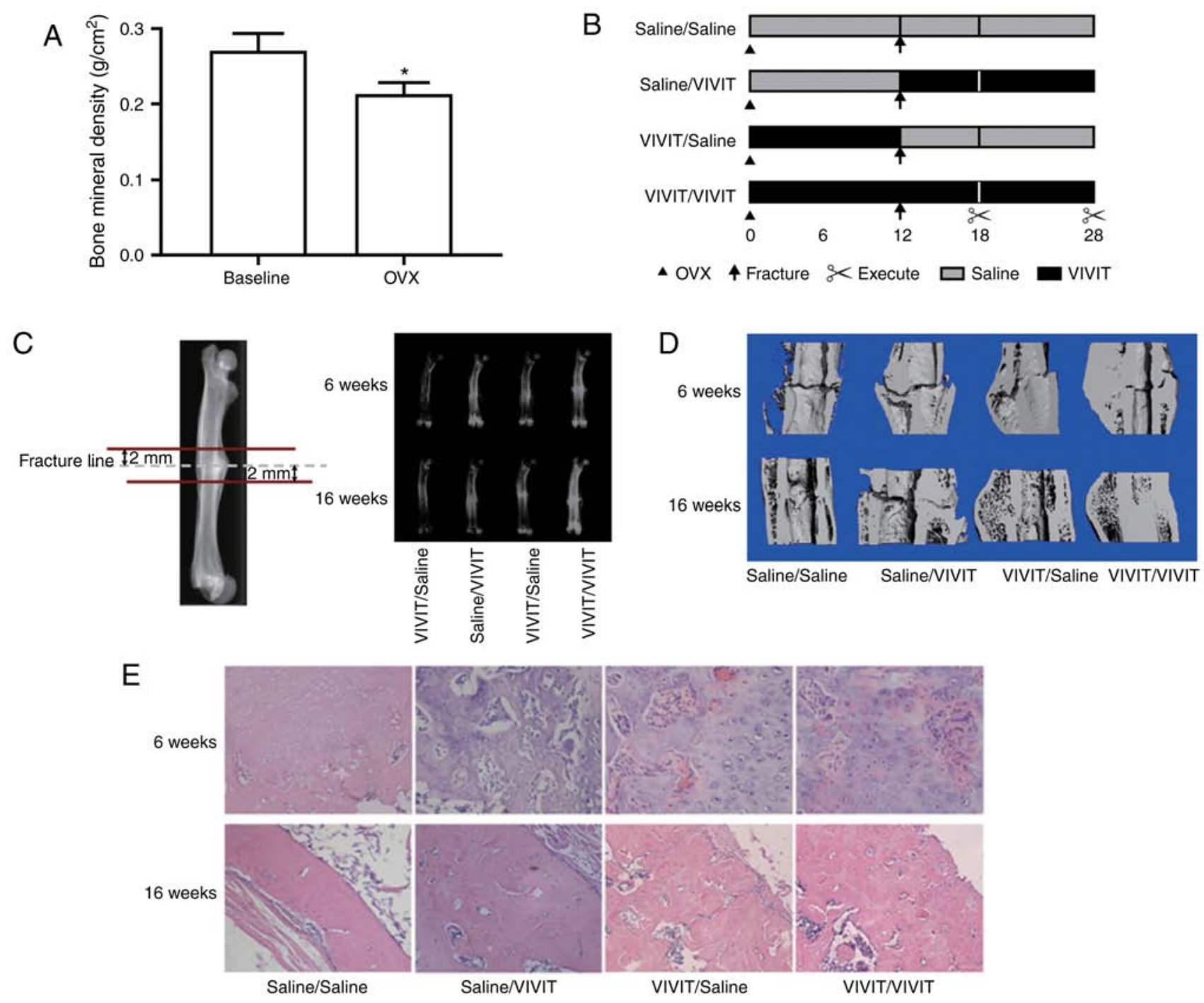

Figure 1. 11R-VIVIT accelerates fracture healing in osteoporotic rats. (A) Bone mineral density. (B) Schematic timeline of the procedures during the study. (C and D) Micro-CT 3D reconstruction. (E) H\&E staining of callus tissue at 6 weeks post-fracture (x200 magnification) and 16 weeks post-fracture (x100 magnification). ${ }^{*} \mathrm{P}<0.05$, compared with baseline.

compared with those treated with saline (Fig. 1C and D) in at 6 and 16 weeks after the fracture. The reconstruction results of micro-CT analysis revealed that at 6- and 16-weeks post-fracture, the 11R-VIVIT-treated rats (particularly the rats from the VIVIT/VIVIT group) exhibited smaller fracture calluses and fracture gaps. At 16 weeks post-fracture, all fracture gaps in the whole-stage 11R-VIVIT-treated rats had almost disappeared, while the other groups exhibited obvious fracture gaps. H\&E staining analysis also revealed that whole-stage 11R-VIVIT treatment increased the formation of bony connective junctions between the fracture gaps, which also suggested that 11R-VIVIT accelerated bone remodeling process in osteoporotic fracture healing (Fig. 1E).

$11 R$-VIVIT increases the osteogenic potential of osteoporotic $B M S C s$. As the osteogenic potential of BMSCs is a key factor during osteoporotic fracture healing, the normal and osteoporotic BMSCs were used to induce osteogenesis in vitro to determine whether 11R-VIVIT would affect the bone formation ability of osteoporotic BMSCs. The results of flow cytometric analysis revealed that the isolated cells were positive for CD73 and CD90, whereas they were negative for $\mathrm{CD} 11 \mathrm{~b}$ and $\mathrm{CD} 45$, indicating that the isolated cells were BMSCs (Fig. 2A). ALP staining, ALP activity assays and Alizarin Red staining were then performed. The osteoporotic BMSCs treated with 11R-VIVIT exhibited more mineralized nodules in the extracellular matrix than the cells from the OVX group (Fig. 2B and D). The results of Alizarin Red S staining revealed that the BMSCs from the OVX group had less calcium deposition than those from the normal group; treatment with11R-VIVIT increased calcium deposition (Fig. 2C and E). The results of semi-quantitative PCR revealed that the normal BMSCs expressed higher levels of ALP, osteocalcin (OCN) and osteopontin (OPN) than the osteoporotic BMSCs (Fig. 2F). The osteoporotic BMSCs were then treated with 11R-VIVIT, at the concentration of 0.1-10 $\mu \mathrm{M}$. ALP staining and Alizarin Red S staining revealed that the ALP activity and the mineralized nodules of the osteogenic BMSCs gradually increased in response to the increasing 11R-VIVIT concentration in a concentration-dependent manner. Semi-quantitative PCR revealed higher expression levels of ALP, OCN and OPN mRNA in response to $11 \mathrm{R}-\mathrm{VIVIT}$ treatment compared with the 
A

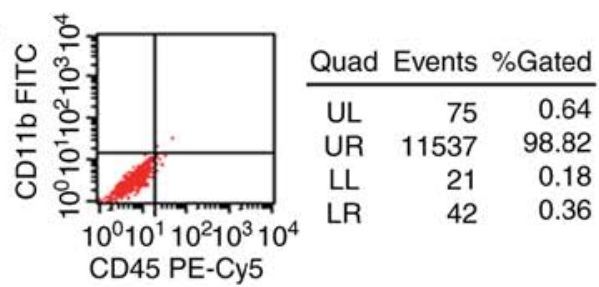

B

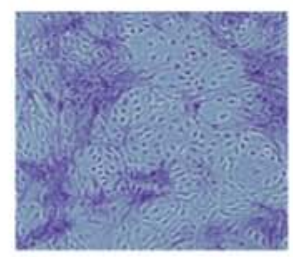

Normal

C

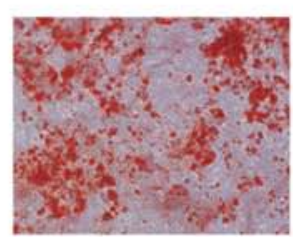

Normal

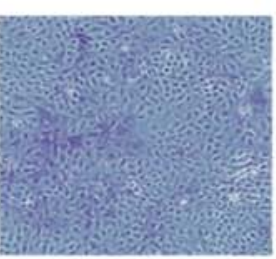

OVX

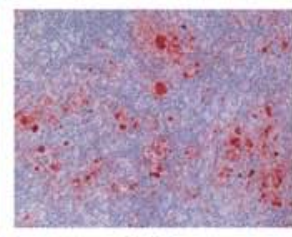

ovX

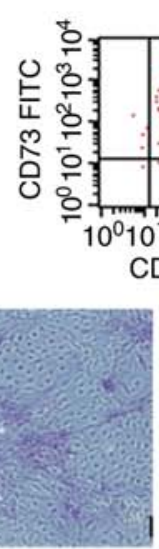

OVX+VIVIT $0.1 \mu \mathrm{M}$

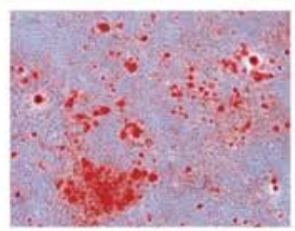

OVX+VIVIT $0.1 \mu \mathrm{M}$

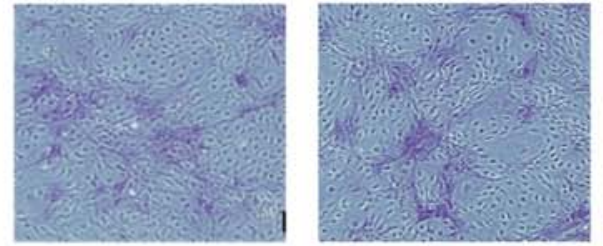

OVX+VIVIT $1 \mu \mathrm{M}$

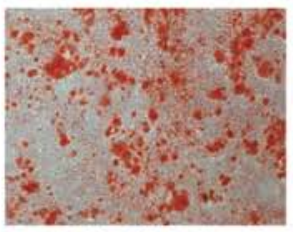

OVX+VIVIT $1 \mu \mathrm{M}$

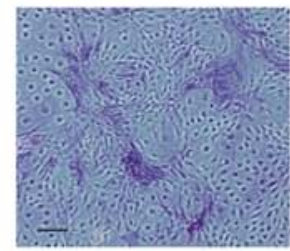

OVX+VIVIT $10 \mu \mathrm{M}$

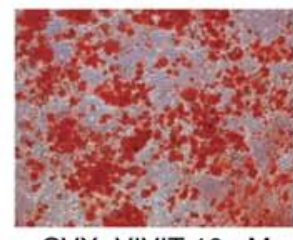

OVX+VIVIT $10 \mu \mathrm{M}$
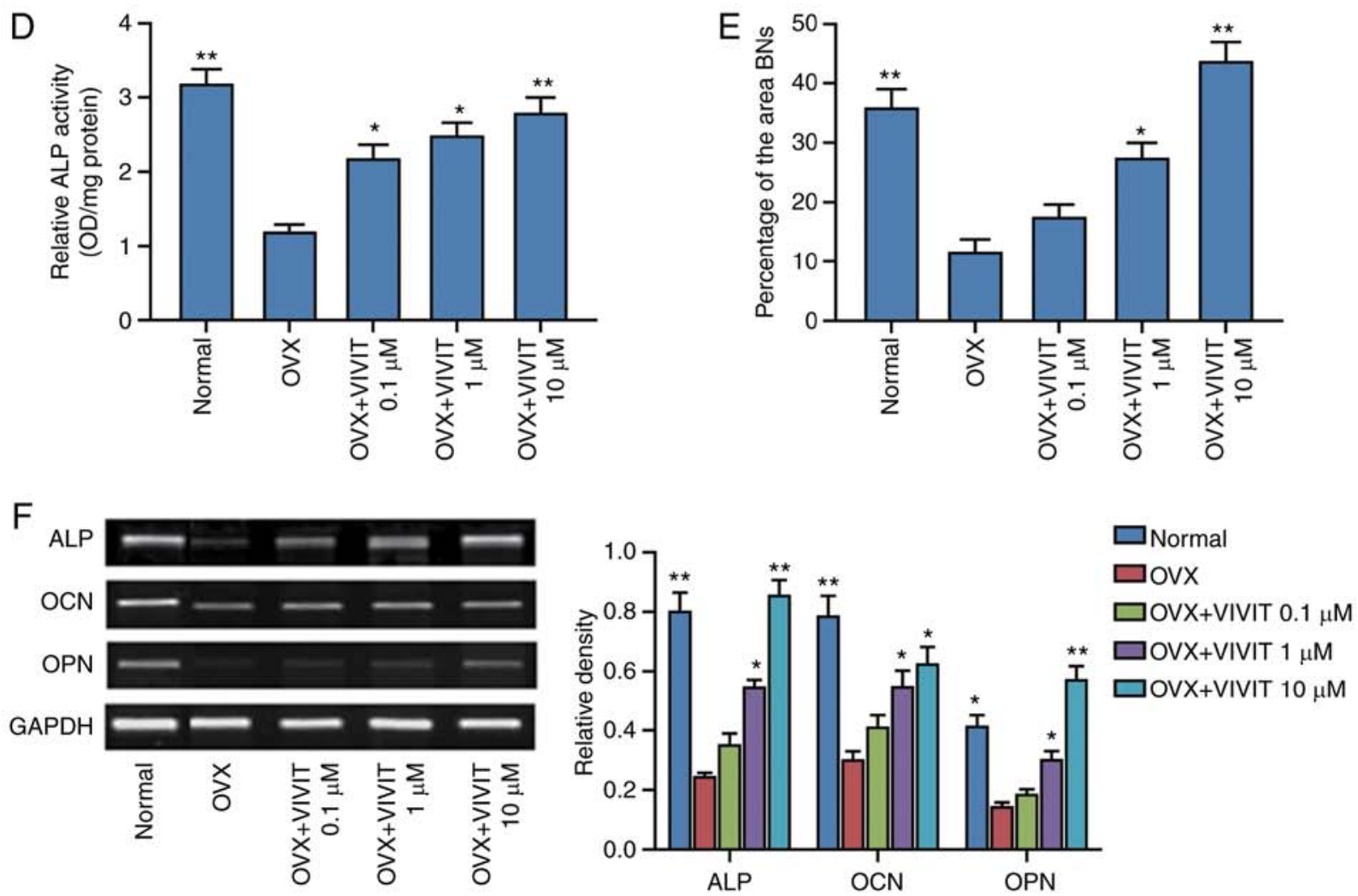

Figure 2. 11R-VIVIT increases the osteogenic potential of osteoporotic BMSCs. (A) Flow cytometric analysis of BMSC surface antigens. (B) ALP staining results. (C) Alizarin Red S staining results. (D) Quantitative results of ALP staining. (E) Quantitative results of Alizarin Red S staining. (F) Semi-quantitative PCR detection of osteogenesis-related gens. ${ }^{*} \mathrm{P}<0.05$ and ${ }^{* *} \mathrm{P}<0.01$, compared with the OVX group. BMSC, bone marrow-derived mesenchymal stem cell; OVX, ovariectomy; ALP, alkaline phosphatase.

OVX control group. Hence, 11R-VIVIT at a concentration of $10 \mu \mathrm{M}$ was employed for further analyses in the present study.

11R-VIVIT impairs the adipogenic potential of osteoporotic BMSCs. As the adipogenic potential of BMSCs also plays vital roles in osteoporotic fracture healing, the normal and osteoporotic BMSCs were used to induce adipogenesis in vitro to determine whether 11R-VIVIT can affect the adipogenic ability of osteoporotic BMSCs. The results of RT-qPCR and Oil Red O staining revealed that the osteoporotic BMSCs had 


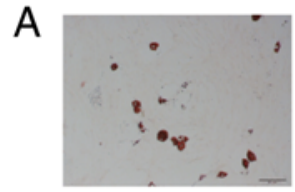

$\mathrm{BMSC}^{\mathrm{Normal}}+\mathrm{AIM}$

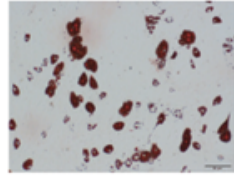

$\mathrm{BMSC}^{\mathrm{OVX}}+\mathrm{AIM}$

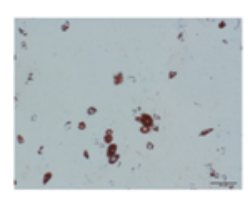

$\mathrm{BMSCOVX}_{+} \mathrm{AIM}+$ 11R-VIVIT
B

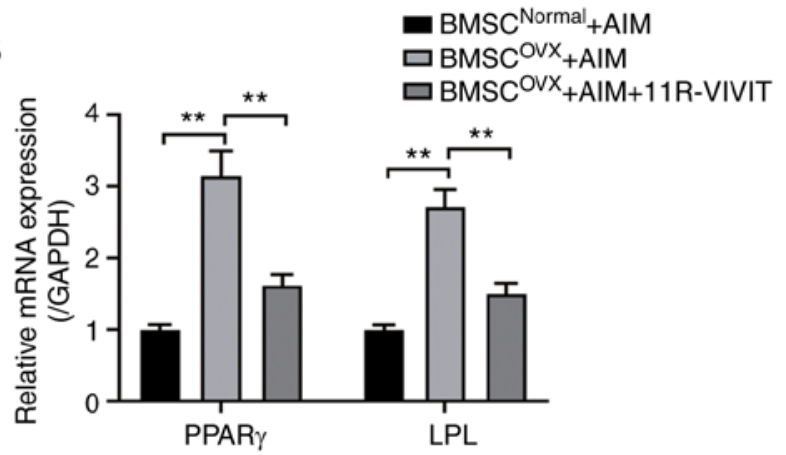

Figure 3. 11R-VIVIT impairs the adipogenic potential of osteoporotic BMSCs. (A) Oil Red O staining results. (B) Following adipogenic induction, the mRNA levels of PPAR $\gamma$ and LPL in BMSCs were detected by RT-qPCR . * ${ }^{*}<0.01$. BMSC, bone marrow-derived mesenchymal stem cell; OVX, ovariectomy; PPAR $\gamma$, peroxisome proliferator-activated receptor $\gamma$; LPL, lipoprotein lipase.
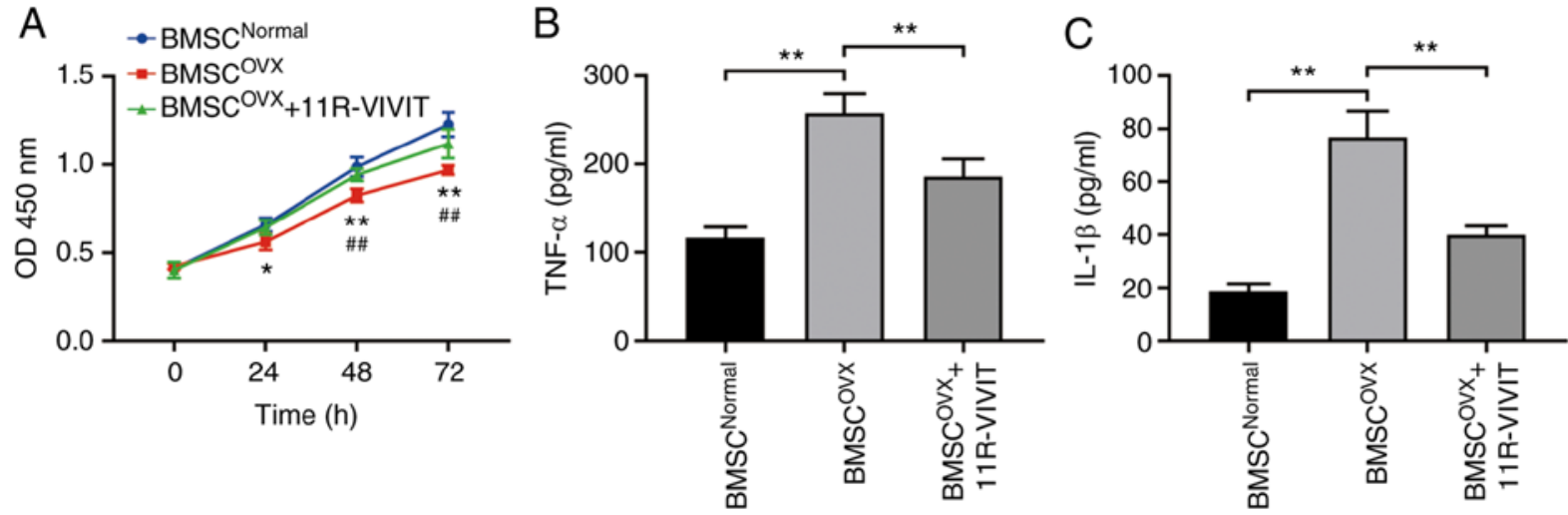

Figure 4. 11R-VIVIT alleviates the inflammatory levels of osteoporotic BMSCs and increased their viability. (A) Cell viability of BMSCs in the OVX group was decreased when compared with the normal group, and 11R-VIVIT treatment relieved the decreased cell viability caused by OVX. ${ }^{*} \mathrm{P}<0.05$ and ${ }^{* *} \mathrm{P}<0.01$, normal group vs. OVX. ${ }^{\# \#} \mathrm{P}<0.01$, OVX vs. OVX + 11R-VIVIT. (B and C) Expression levels of the inflammatory factors, TNF- $\alpha$ and IL-1 $\beta$, in BMSCs in the OVX group were increased, and 11R-VIVIT relieved the increase in the levels of TNF- $\alpha$ and IL-1 $\beta$ induced by OVX. ${ }^{* *} \mathrm{P}<0.01$. BMSC, bone marrow-derived mesenchymal stem cell; OVX, ovariectomy; TNF- $\alpha$, tumor necrosis factor $\alpha$; IL-1 $\beta$, interleukin $1 \beta$.

a greater adipogenic capacity than the normal BMSCs (Fig. 3). After the osteoporotic BMSCs were treated with 11R-VIVIT, Oil Red $\mathrm{O}$ staining revealed that the adipogenic activity of the osteoporotic BMSCs was inhibited (Fig. 3A). Likewise, the results of RT-qPCR revealed lower mRNA expression levels of peroxisome proliferator-activated receptor (PPAR) $\gamma$ and lipoprotein lipase (LPL) in response to 11R-VIVIT treatment compared with the OVX control group (Fig. 3B).

$11 R$-VIVIT attenuates the inflammatory levels of osteoporotic BMSCs and increases cell viability. ELISA and CCK-8 assay were used to determine whether 11R-VIVIT can affect the viability and inflammatory levels of osteoporotic BMSCs. CCK-8 assay revealed that the viability of osteoporotic BMSCs was markedly lower than that of normal BMSCs. In addition, 11R-VIVIT treatment enhanced the viability of osteoporotic BMSCs compared with the BMSCs from the OVX group (Fig. 4A). Finally, the results of ELISA revealed a same pattern as CCK-8 assay. In detail, 11R-VIVIT treatment eliminated the levels of inflammatory factors in osteoporotic BMSCs (Fig. 4B and C).

$11 R$-VIVIT promotes autophagy by inactivating AKT/NFATcI signaling. Subsequently, western blot analysis was performed to determine whether autophagy is involved in the reversion of osteogenic capacity under 11R-VIVIT treatment. The results revealed a significant downregulation in the LC3-II/LC3-I ratio and the upregulation of p62 protein expression in osteoporotic BMSCs during osteogenesis compared with the normal ones, which indicated that osteoporosis impaired the autophagy of BMSCs. By contrast, a significant upregulation in the LC3-II/LC3-I ratio and the downregulation of p62 activity were observed in response to treatment with 11R-VIVIT (Fig. 5A), suggesting that 11R-VIVIT acted as an activator of autophagy in osteoporotic BMSCs.

The present study then attempted to elucidate the molecular mechanisms through which 11R-VIVIT led to the enhancement of autophagy and the osteogenesis of osteoporotic BMSCs. As the AKT/GSK-3 $\beta$ signaling pathway plays a vital role in the autophagy and osteogenic differentiation of BMSCs, the present study determined whether there was a crosstalk between 11R-VIVIT and AKT/GSK-3 $\beta$ signaling. Western blot analysis revealed a significant upregulation of $\mathrm{p}-\mathrm{AKT}$ and $\mathrm{p}-\mathrm{GSK}-3 \beta$ in osteoporotic BMSCs during osteogenesis compared with the normal BMSCs (Fig. 5B). Thereafter, the osteoporotic BMSCs were treated with 11R-VIVIT, which resulted in the downregulation of $\mathrm{p}$-AKT in osteoporotic BMSCs, while the level of p-GSK-3 $\beta$ exhibited no significant changes in the osteoporotic BMSCs. In order to confirm these results, LY294002 was used to 

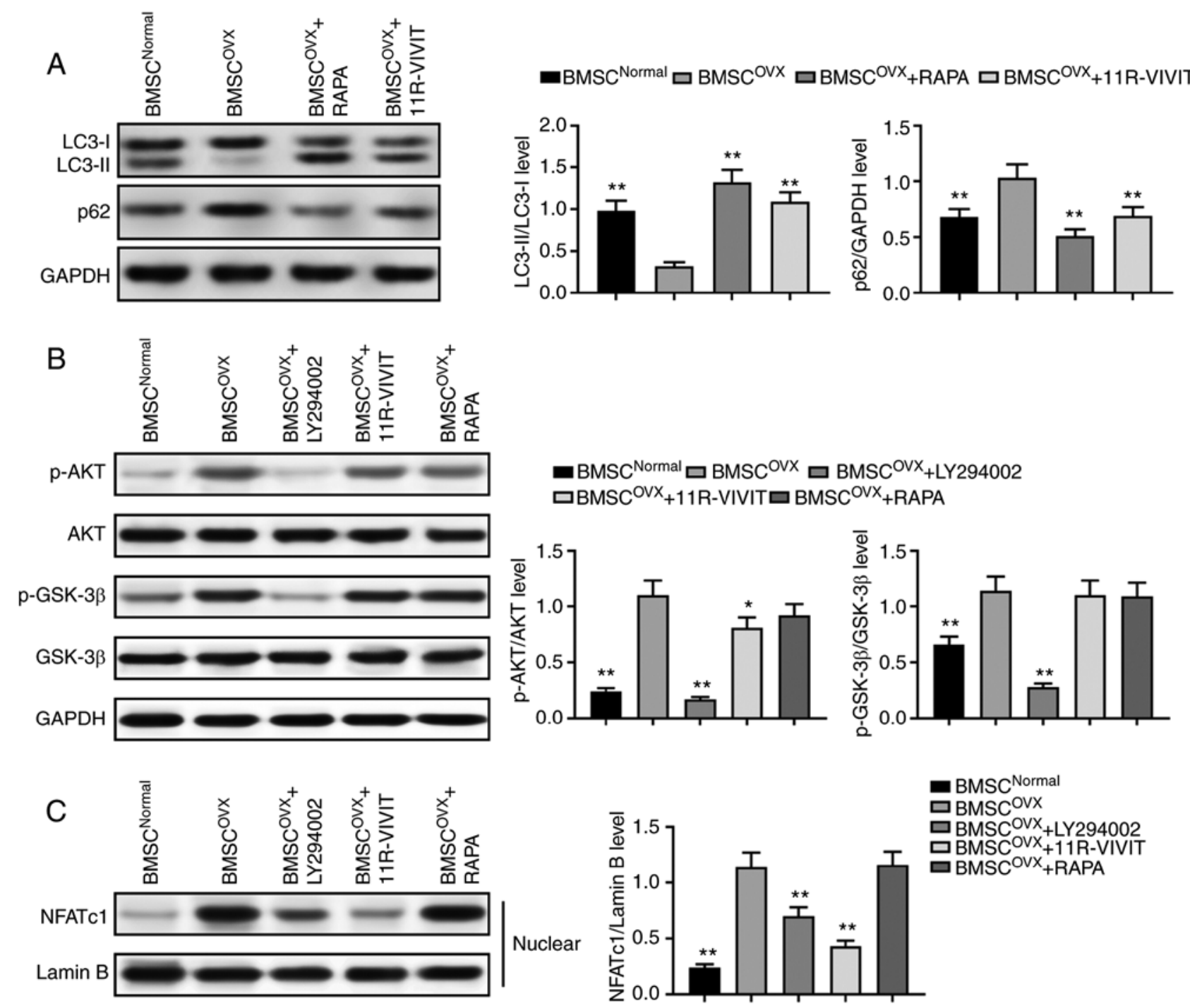

Figure 5. 11R-VIVIT activates autophagy by activating AKT/GSK-3 $\beta$ signaling. (A) Western blot analysis of the autophagy-related proteins, LC3-I/II and p62. The autophagy level in BMSCs from rats subjected to OVX was inhibited, and 11R-VIVIT promoted autophagy. (B) Western blot analysis of AKT and GSK-3 $\beta$ proteins. (C) Following osteogenic induction, the level of NFTAc1 in the nucleus of BMSCs in the OVX group was higher than that in the normal group. LY294002 and 11R-VIVIT inhibited the entry of NFTAc1 into the nucleus. ${ }^{*} \mathrm{P}<0.05$ and ${ }^{* *} \mathrm{P}<0.01$, compared with BMSCs from rats subjected to OVX. BMSC, bone marrow-derived mesenchymal stem cell; OVX, ovariectomy; AKT, protein kinase B; GSK-3 $\beta$, glycogen synthase kinase $3 \beta$; RAPA, rapamycin.

inhibit AKT signaling and rapamycin (RAPA) was used to activate autophagy. Western blot analysis revealed that the activation of autophagy and the inhibition of AKT signaling exerted similar effects as observed with 11R-VIVIT treatment. Finally, western blot analysis revealed that the nuclear expression of NFATc1 was significantly increased in osteoporotic BMSCs compared with the normal ones. The nuclear expression of NFATc1 was decreased in the 11R-VIVIT treatment group (Fig. 5C), indicating that 11R-VIVIT inhibited the activation of NFATc1. Combined with AKT expression inhibition and autophagy activation, these results demonstrated that $11 \mathrm{R}-$ VIVIT activated autophagy and inhibited NFATc1 activation by inactivating AKT expression.

Subsequently, Alizarin Red S and ALP staining were performed to confirm the osteogenic potential of the BMSCs after the various treatments. The osteoporotic BMSCs treated with 11R-VIVIT, LY294002 and RAPA exhibited an increased osteogenic potential when compared with the osteoporotic BMSCs without treatment (Fig. 6A-D). Western blot analysis of Runx 2 and ALP also revealed that the activation of autophagy and the inhibition of AKT signaling exerted similar effects as those observed with 11R-VIVIT treatment (Fig. 6E).

\section{Discussion}

The most salient finding of the present study was that the inhibition of NFATc1 with 11R-VIVIT significantly enhanced osteoporotic fracture healing. In addition, the present study also revealed that 11R-VIVIT treatment impaired adipogenesis and enhanced the osteoblast differentiation of the osteoporotic BMSCs for the first time. Furthermore, the defective osteogenic phenotype was confirmed in osteoporotic BMSCs, which was reversed by 11R-VIVIT treatment. Moreover, 11R-VIVIT acted as an activator of autophagy in osteoporotic BMSCs. Taken together, the findings indicated that 11R-VIVIT promoted autophagy and osteoblast differentiation for fracture healing through regulating the AKT/NFATc1 signaling pathway.

Osteoblasts are essential for osteoporotic fracture healing $(3,22)$. During this complex and dynamic process, the 
A

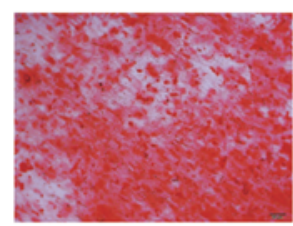

BMSC $^{\text {Normal }}+$ OIM

B

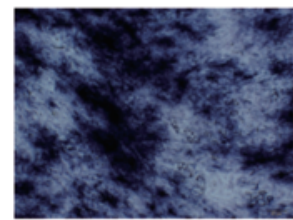

BMSC $^{\text {Normal }}+{ }_{+}$IM

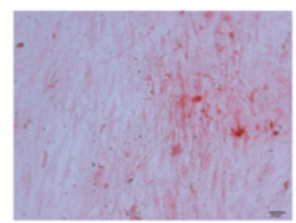

$\mathrm{BMSC}^{\mathrm{OVX}}+\mathrm{OIM}$

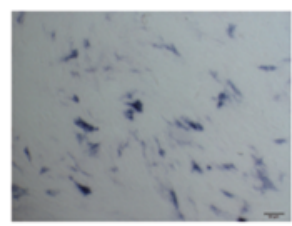

$\mathrm{BMSC}^{\mathrm{OVX}}+\mathrm{OIM}$

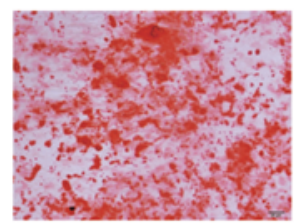

BMSC $^{\text {OVX }}+$ OIM+LY294002

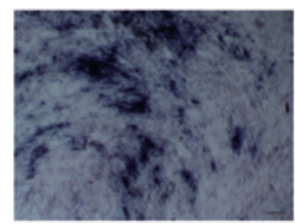

$\mathrm{BMSC}^{\mathrm{OVX}}+\mathrm{OIM}+\mathrm{LY} 294002$

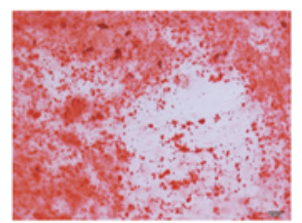

$\mathrm{BMSC}^{\mathrm{OVX}}+\mathrm{OIM}+11 \mathrm{R}$-VIVIT

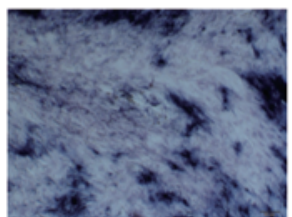

$\mathrm{BMSC}^{\mathrm{OVX}}+\mathrm{OIM}+11 \mathrm{R}-\mathrm{VIVIT}$

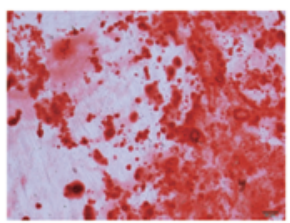

$\mathrm{BMSC}^{\text {OVX }}+\mathrm{OIM+RAPA}$

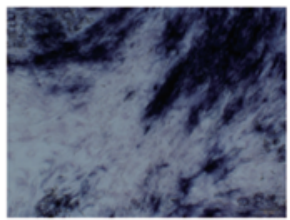

$\mathrm{BMSC}^{\mathrm{OVX}}+\mathrm{OIM}+\mathrm{RAPA}$
C

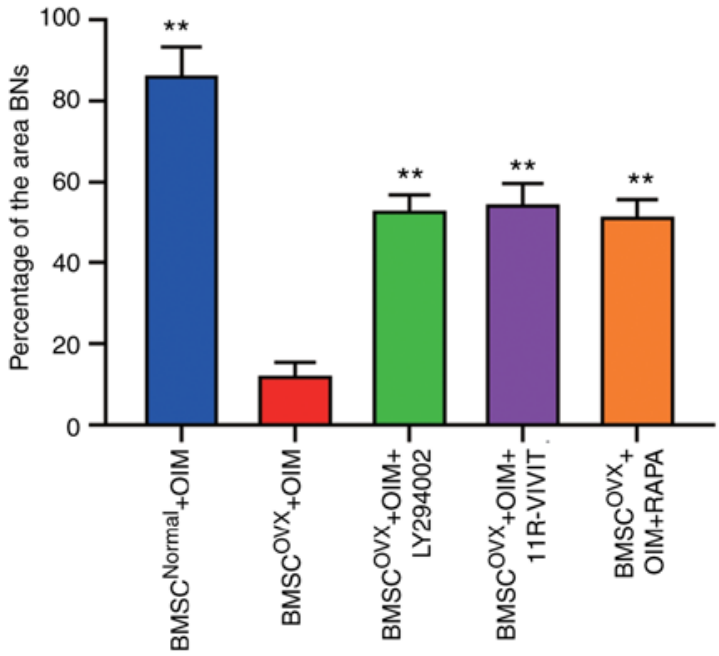

D

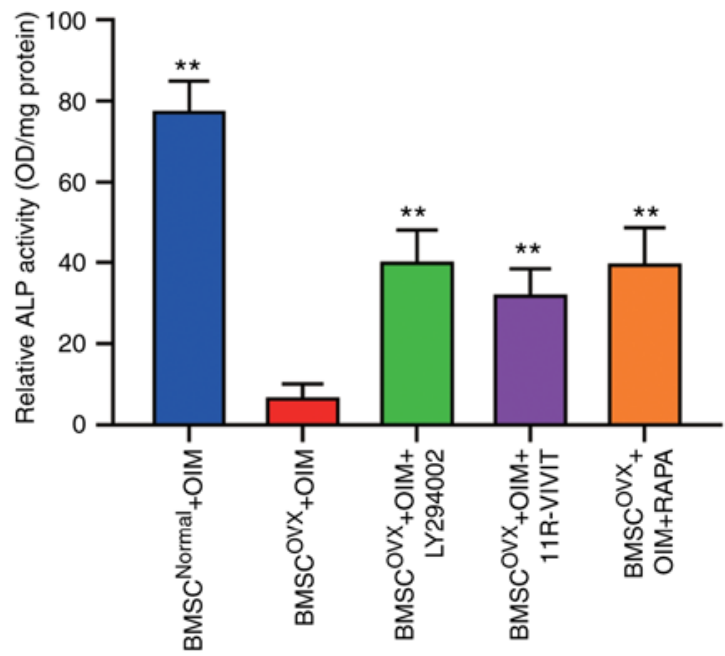

E

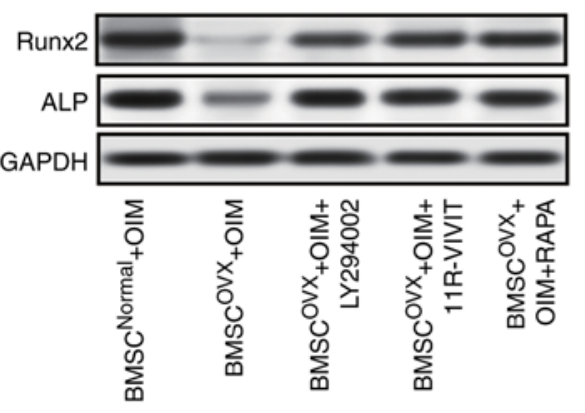

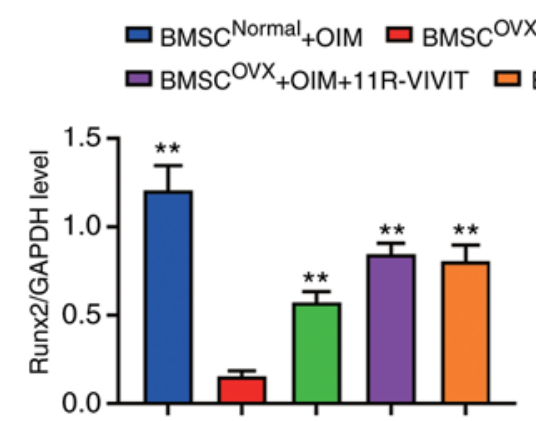

口 $\mathrm{BMSC}^{\mathrm{Normal}}+\mathrm{OIM}$ 口 $\mathrm{BMSC}^{\mathrm{OVX}}+\mathrm{OIM}$ 口 $\mathrm{BMSC}^{\mathrm{OVX}}+\mathrm{OIM}+\mathrm{LY} 294002$
口 $\mathrm{BMSC}^{\mathrm{OVX}}+\mathrm{OIM}+11 \mathrm{R}-\mathrm{VIVIT}$ 口 $\mathrm{BMSC}^{\mathrm{OVX}}+\mathrm{OIM}+\mathrm{RAPA}$

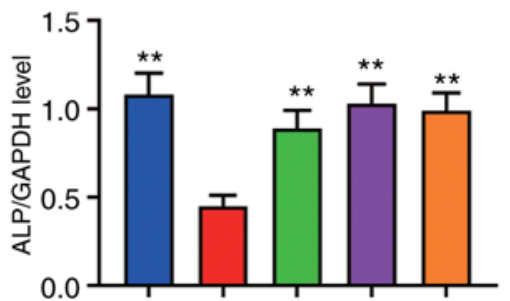

Figure 6. AKT/NFATc1 signaling pathway regulates the osteogenic differentiation of BSMCs. (A) ALP staining results. (B) Alizarin Red S staining results. (C) Quantitative results of ALP staining. (D) Quantitative results of Alizarin Red S staining. (E) Following osteogenic induction, the levels of osteogenic marker genes Runx2 and ALP of BMSCs in the OVX group were significantly reduced, while LY294002 and 11R-VIVIT promoted their expression. ${ }^{* *} \mathrm{P}<0.01$, compared with BMSCs from rats subjected to OVX. BMSC, bone marrow-derived mesenchymal stem cell; OVX, ovariectomy; ALP, alkaline phosphatase; AKT, protein kinase B; GSK-3 $\beta$, glycogen synthase kinase $3 \beta$; NFAT, nuclear factor of activated T-cells; RAPA, rapamycin.

disorganized differentiation of BMSCs will lead to abnormal fracture healing, even fracture non-unions. The replacement of osteoblastogenic activity by adipogenic activity is a typical feature of osteoporotic BMSCs, in which osteoblast formation is impaired to a large extent. Therefore, the rescue of the impaired osteogenic phenotype of osteoporotic BMSCs is helpful for promoting bone formation and accelerating fracture healing (23-25). In the present it was demonstrated that osteoporotic rats with fractures exhibited evidently impaired fracture healing at 6 and 16 weeks after the fracture, when treated with saline alone. Furthermore, rats treated with whole-stage 11R-VIVIT exhibited an improved fracture healing process.

NFATc1 is regulated by calmodulin/ $\mathrm{Ca}^{2+}$-dependent signaling. Activated $\mathrm{Cn}$ dephosphorylates NFATcl protein and leads to its rapid nuclear translocation. Likewise, the excessive activation of NFATc1 is capable of by passing the requirement for RANKL and inducing osteoclast differentiation. The selective inhibition of NFATc1 activation has been shown to result in impaired osteoclast formation and reduced bone-resorption (26). Furthermore, it has been reported that NFATc1 signaling negatively regulates the formation of 
osteoprogenitor cells (11). The selective blocking of NFATc1 signaling also favors osteoblast and bone formation $(11,12)$, which indicates the potential of 11R-VIVIT in regulating bone resorption and fracture unions. 11R-VIVIT has been reported to inhibit NFAT directly and to prevent the nuclear translocation of NFAT and cell apoptosis (27). Consistently, in the present study, 11R-VIVIT led to the significant inhibition of NFATc1 expression and nuclear translocation. Furthermore, the results revealed that 11R-VIVIT stimulated bone formation and attenuated osteoporosis by decreasing NFATc1 expression and the expression of inflammation-related molecules, which was in line with the results of the study by Li et al (10).

Previous findings have demonstrated that the inactivation of NFATc1 by 11R-VIVIT enhances osteoblast differentiation (28), which is in accordance with the results of the present study that 11R-VIVIT enhanced the osteogenic differentiation of osteoporotic BMSCs by dysregulating the AKT/NFATc1 signaling pathway to promote the fracture healing. The results also revealed that 11R-VIVIT treatment restored the viability of osteoporotic BMSCs and decreased osteoporosis-induced inflammation. In addition, the results revealed that11R-VIVIT acted as an activator of autophagy in osteoporotic BMSCs. 11R-VIVIT also resulted in the downregulation of p-AKT in osteoporotic BMSCs, similar to the effects of LY294002 and rapamycin. Furthermore, previous research has demonstrated that $11 \mathrm{R}-\mathrm{VIVIT}$ is a protective agent against a number of pathological conditions. Cai et al (29) reported that the inhibition of NFATc1 using 11R-VIVIT significantly reduced the development of nerve injury-induced tactile allodynia. Taken together, the results of the present study established 11R-VIVIT as a potential therapeutic strategy against several common diseases, including osteoporotic fractures.

However, certain limitations to the present study should be mentioned. Firstly, only limited autophagy-related proteins were detected due to limited time and attention. Further autophagy-related proteins need to be detected in the future for the better understanding of the association between 11R-VIVIT and autophagy, as well as cell autophagy and the osteogenic differentiation of osteoporotic BMSCs. Furthermore, other signaling pathways, such as inflammation-related signaling pathways need to be examined. Thus, further studies are required for the better elucidation of the link between 11R-VIVIT and osteoporotic fracture healing.

In conclusion, the present findings demonstrated that 11R-VIVIT enhanced rat osteoporotic fracture healing by modifying AKT/NFATc1 signaling. Moreover, 11R-VIVIT may also be responsible for promoting the shift of osteoporotic BMSCs to an osteoblastic phenotype, and may promote bone formation during osteoporosis.

\section{Acknowledgements}

Not applicable.

\section{Funding}

This study was supported by the Hangzhou Science and Technology Development Project (grant no. 20191203B87).

\section{Availability of data and materials}

The datasets used and/or analyzed during the current study are available from the corresponding author on reasonable request.

\section{Authors' contributions}

$\mathrm{CH}$ designed the study, performed the experiments, collected and analyzed data and wrote the manuscript. XW, WJ, ZB and LZ performed the experiments, and collected and analyzed data. ML designed the study, conducted experiments and was involved in manuscript preparation. $\mathrm{CH}$ and ML confirm the authenticity of all the raw data. All authors have read and approved the final manuscript.

\section{Ethics approval and consent to participate}

All animal experiments were approved by the Institutional Animal Care and Use Committee of The Affiliated Hangzhou First People's Hospital, Hangzhou, China (no. ZJCLA-IACUC-20080010).

\section{Patient consent for publication}

Not applicable.

\section{Competing interests}

The authors declare that they have no competing interests.

\section{References}

1. Liang W, Zhuo X, Tang Z, Wei X and Li B: Calcitonin gene-related peptide stimulates proliferation and osteogenic differentiation of osteoporotic rat-derived bone mesenchymal stem cells. Mol Cell Biochem 402: 101-110, 2015.

2. Zhang X, Wang Y, Zhao H, Han X, Zhao T, Qu P, Li G and Wang W: Extracellular vesicle-encapsulated miR-22-3p from bone marrow mesenchymal stem cell promotes osteogenic differentiation via FTO inhibition. Stem Cell Res Ther 11: 227, 2020.

3. Ali D, Chen L, Kowal JM, Okla M, Manikandan M, AlShehri M, AlMana Y, AlObaidan R, AlOtaibi N, Hamam R, et al: Resveratrol inhibits adipocyte differentiation and cellular senescence of human bone marrow stromal stem cells. Bone 133: $115252,2020$.

4. An Q, Wu D, Ma Y, Zhou B and Liu Q: Suppression of Evil promotes the osteogenic differentiation and inhibits the adipogenic differentiation of bone marrow-derived mesenchymal stem cells in vitro. Int J Mol Med 36: 1615-1622, 2015.

5. Wang Z, Wang D, Yang D, Zhen W, Zhang J and Peng S: The effect of icariin on bone metabolism and its potential clinical application. Osteoporos Int 29: 535-544, 2018.

6. Chen X, Zhi X, Cao L, Weng W, Pan P, Hu H, Liu C, Zhao Q, Zhou Q, Cui J and Su J: Matrine derivate MASM uncovers a novel function for ribosomal protein S5 in osteoclastogenesis and postmenopausal osteoporosis. Cell Death Dis 8: e3037, 2017.

7. Mesner LD, Calabrese GM, Al-Barghouthi B, Gatti DM, Sundberg JP, Churchill GA, Godfrey DA, Ackert-Bicknell CL and Farber CR: Mouse genome-wide association and systems genetics identifies Lhfp as a regulator of bone mass. PLoS Genet 15: e1008123, 2019.

8. Zhang Y, Wei L, Chang J, Miron RJ, Shi B, Yi S and Wu C: Strontium-incorporated mesoporous bioactive glass scaffolds stimulating in vitro proliferation and differentiation of bone marrow stromal cells and in vivo regeneration of osteoporotic bone defects. J Mater Chem B 1: 5711-5722, 2013.

9. He LH, Liu M, He Y, Xiao E, Zhao L, Zhang T, Yang HQ and Zhang Y: TRPV1 deletion impaired fracture healing and inhibited osteoclast and osteoblast differentiation. Sci Rep 7: 42385, 2017. 
10. Li M, Wang X, Bian Z, Yao W, He Q, Tian F, Zhang J and Zhu L: Peptide 11R-VIVIT stimulates osteoblastogenesis through regulating the expression of nuclear factor of activated $\mathrm{T}$ cells cytoplasmic 1. Cell Mol Biol (Noisy-le-grand) 63: 46-52, 2017.

11. Boise LH, Petryniak B, Mao X, June CH, Wang CY, Lindsten T, Bravo R, Kovary K, Leiden JM and Thompson CB: The NFAT-1 DNA binding complex in activated T cells contains Fra-1 and JunB. Mol Cell Biol 13: 1911-1919, 1993.

12. Mulero MC, Aubareda A, Orzaez M, Messeguer J, Serrano-Candelas E, Martínez-Hoyer S, Messeguer A, Pérez-Payá E and Pérez-Riba M: Inhibiting the calcineurin-NFAT (nuclear factor of activated T cells) signaling pathway with a regulator of calcineurin-derived peptide without affecting general calcineurin phosphatase activity. J Biol Chem 284: 9394-9401, 2009.

13. Li R, Zhang L, Shi W, Zhang B, Liang X, Liu S and Wang W: NFAT2 mediates high glucose-induced glomerular podocyte apoptosis through increased Bax expression. Exp Cell Res 319: 992-1000, 2013.

14. Yang L, Guan H, He J, Zeng L, Yuan Z, Xu M, Zhang W, Wu X and Guan J: VEGF increases the proliferative capacity and eNOS/NO levels of endothelial progenitor cells through the calcineurin/NFAT signalling pathway. Cell Biol Int 36: 21-27, 2012.

15. Zhang L, Li R, Shi W, Liang X, Liu S, Ye Z, Yu C, Chen Y, Zhang B, Wang W, et al: NFAT2 inhibitor ameliorates diabetic nephropathy and podocyte injury in $\mathrm{db} / \mathrm{db}$ mice. $\mathrm{Br}$ J Pharmacol 170: 426-439, 2013.

16. Sophocleous A and Idris AI: Rodent models of osteoporosis. Bonekey Rep 3: 614, 2014.

17. Fathi E, Valipour B, Sanaat Z, Nozad Charoudeh H and Farahzadi R: Interleukin- $6,-8$, and TGF- $\beta$ secreted from mesenchymal stem cells show functional role in reduction of telomerase activity of leukemia cell via Wnt5a/ $\beta$-catenin and P53 pathways. Adv Pharm Bull 10: 307-314, 2020.

18. Fathi E, Farahzadi R, Valipour B and Sanaat Z: Cytokines secreted from bone marrow derived mesenchymal stem cells promote apoptosis and change cell cycle distribution of K562 cell line as clinical agent in cell transplantation. PLoS One 14: e0215678, 2019

19. Farahzadi R, Fathi E and Vietor I: Mesenchymal stem cells could be considered as a candidate for further studies in cell-based therapy of Alzheimer's disease via targeting the signaling pathways. ACS Chem Neurosci 11: 1424-1435, 2020.

20. Livak KJ and Schmittgen TD: Analysis of relative gene expression data using real-time quantitative PCR and the 2(-Delta Delta C(T)) method. Methods 25: 402-408, 2001.
21. Fathi E, Farahzadi R, Javanmardi S and Vietor I: L-carnitine extends the telomere length of the cardiac differentiated CD117(+)-expressing stem cells. Tissue Cell 67: 101429, 2020.

22. Xia WF, Jung JU, Shun C, Xiong S, Xiong L, Shi XM, Mei L and Xiong WC: Swedish mutant APP suppresses osteoblast differentiation and causes osteoporotic deficit, which are ameliorated by N-acetyl-L-cysteine. J Bone Miner Res 28: 2122-2135, 2013.

23. Zhou Y, Zhu ZL, Guan XX, Hou WW and Yu HY: Reciprocal roles between caffeine and estrogen on bone via differently regulating cAMP/PKA pathway: The possible mechanism for caffeine-induced osteoporosis in women and estrogen's antagonistic effects. Med Hypotheses 73: 83-85, 2009.

24. Xu R, Fu Z, Liu X, Xiao T, Zhang P, Du Y, Yuan H, Cheng J and Jiang H: Transplantation of osteoporotic bone marrow stromal cells rejuvenated by the overexpression of SATB2 prevents alveolar bone loss in ovariectomized rats. Exp Gerontol 84: 71-79, 2016

25. Yu F, Wu F, Li F, Liao X, Wang Y, Li X, Wang C, Shi Y and Ye L: Wnt7b-induced Sox11 functions enhance self-renewal and osteogenic commitment of bone marrow mesenchymal stem cells. Stem Cells 38: 1020-1033, 2020.

26. Crotti TN, Flannery M, Walsh NC, Fleming JD, Goldring SR and McHugh KP: NFATc1 regulation of the human beta3 integrin promoter in osteoclast differentiation. Gene 372: 92-102, 2006.

27. Bodmer D, Perkovic A, Sekulic-Jablanovic M, Wright MB and Petkovic V: Pasireotide prevents nuclear factor of activated T cells nuclear translocation and acts as a protective agent in aminoglycoside-induced auditory hair cell loss. J Neurochem 139: 1113-1123, 2016

28. Maoqiang L, Zhenan Z, Fengxiang L, Gang W, Yuanqing M, Ming L, Xin Z and Tingting T: Enhancement of osteoblast differentiation that is inhibited by titanium particles through inactivation of NFATc1 by VIVIT peptide. J Biomed Mater Res A 95: 727-734, 2010

29. Cai YQ, Chen SR and Pan HL: Upregulation of nuclear factor of activated T-cells by nerve injury contributes to development of neuropathic pain. J Pharmacol Exp Ther 345: 161-168, 2013.

This work is licensed under a Creative Commons Attribution-NonCommercial-NoDerivatives 4.0 International (CC BY-NC-ND 4.0) License. 